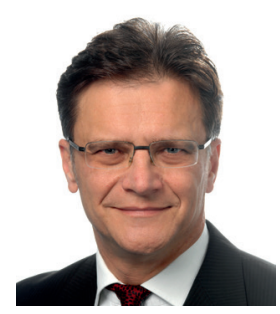

Prof. Dr. Dr. Rudolf Hatz r.hatz@asklepios.com

\title{
Im Fokus: Pleuraerkrankungen - aktuelle Diagnostik und Therapie
}

Imzweiten Themenheft Pneumologievon KaRGer Kompass stehen die für die alltägliche Praxis so wichtigen Pleuraerkrankungen, der Pleuraerguss und der Pneumothorax im Mittelpunkt. Klinisch präsentieren sie sich oft als sekundär akut symptomatische Folgezustände anderer primärer Grunderkrankungen, die einer sofortigen diagnostischen Abklärung und Behandlung bedürfen. Zwei ausgewählten Übersichtsarbeiten berichten hier über den neuesten Stand der empfohlenen diagnostischen und therapeutischen Möglichkeiten beider Krankheitsentitäten.

In Deutschland liegt die jährliche Inzidenz des malignen Pleuraergusses bei ca. 56000. In vielen Fällen ist die Pleurapunktion unter sonographischer Kontrolle und sachgerechter Aufarbeitung diagnostisch zielführend und führt bei großen Ergussmengen zur therapeutischen Entlastung. Auch der Einsatz von getunnelten Pleurakathetern ist hier eine Behandlungsoption, wie Dr. Franz Stanzel im Wissenstransfer auf S. 68 darstellt.

Bleibt die Ursache des Ergusses unklar und/oder können häufige Rezidive und damit die führende Symptomatik wie Dyspnoe und thorakale Schmerzen nicht behoben werden, ist die Anwendung minimal invasiver Techniken wie die internistische Thorakoskopie (auch Pleuroskopie genannt) oder die chirurgische videoassistierte Thorakoskopie (VATS) indiziert. Insbesondere beim malignen Erguss (Pleurakarzinose, Pleuramesotheliom) führen diese Maßnahmen zu einer sehr effizienten und raschen Diagnose und definitiven palliativen Therapie (Talkum).

Der primäre Spontanpneumothorax (PSP) stellt ebenfalls ein häufiges klinisch sehr relevantes Problem dar, seine Inzidenz liegt hierzulande bei 8-9/100000 Einwohner. Die Therapiemöglichkeiten reichen von der «Wait-and-see»-Taktik bei kleinen Randpneus über die weniger invasiven $\mathrm{Na}$ delaspiration oder Bülau-Drainagen-Anlage beim Erstereignis bis zur minimal invasiven Pleuroskopie in Lokalanästhesie oder chirurgischen VATS in Allgemeinnarkose und Doppellumenintubation bei mehrtägiger Persistenz (4-7 Tage) des Pneumothorax bzw. bei Auftreten eines Rezidivs. Der gemeinsame therapeutische Nenner in der minimal invasiven Behandlung beider Krankheitsentitäten im therapierefraktären Stadium oder beim Rezidiv ist das rasche Herbeiführen einer suffizienten und dauerhaften Pleurodese, um weitere Komplikationen der Erkrankungen zu vermeiden und damit eine schnellere Symptomfreiheit für den Patienten zur erreichen. Die internistische und chirurgische Thorakoskopie stellen bei richtiger Indikationsstellung, ausreichender Erfahrung der behandelnden Kollegen und sorgfältiger Abwägung der Vor- und Nachteile des jeweiligen Verfahrens den Goldstandard in der Diagnostik und Therapie pleuraler Erkrankungen dar.

Darüber hinaus bietet KaRger Kompass PNeUmologie ein thematisch breites Spektrum ausgesuchter Artikel aus RESPIRATION, die durch die Kommentierung der Experten einen direkten Wissenstransfer in die Praxis erlauben. Der Leser kann sich somit effektiv und gleichzeitig umfassend über aktuelle Entwicklungen in der Pneumologie informieren.

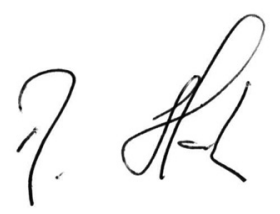

\section{KARGER \\ Fax +497614520714 \\ information@karger.com} www.karger.com
() 2013 S. Karger GmbH, Freiburg

$2296-0368 / 13 / 0012-0053 \$ 38.00 / 0$

Accessible online at:

www.karger.com/kkp
Prof. Dr. Dr. Rudolf Hatz

Klinik für Thoraxchirurgie

Lungenfachkliniken Gauting, Klinikum der Universität München

Robert-Koch-Allee 2, 82131 Gauting (Deutschland) 